\title{
Transient and steady-state heat conduction analysis of two-dimensional functionally graded materials using particle method
}

\author{
H. Sakurai \\ Department of Design and Computer Applications, \\ Miyagi National College of Technology, Japan
}

\begin{abstract}
The moving-particle semi-implicit (MPS) method, one of the particle methods, is an effective numerical simulation method for incompressible inviscid flows with free surfaces. The discretization scheme of the particle method can be utilized for discretizations of gradient, divergence and Laplace operators in the partial differential equations such as the diffusion equation and the wave equation. In recent years, advanced materials known as Functionally Graded Materials (FGMs) have drawn considerable interest. These FGMs are expected to be highly heat resistant materials that can be used under high temperature and high temperature gradient conditions. Because of this, it is important to investigate the temperature distributions in the FGMs. The purpose of this work is to present a numerical analysis of the heat conduction for two-dimensional FGMs where the thermal conductivity is a function of the spatial coordinates using the particle method. Analytical solutions and finite element solutions are compared with the present results, and the validity of the present method is shown.
\end{abstract}

Keywords: particle method, functionally graded materials, heat conduction, transient analysis, steady-state analysis.

\section{Introduction}

In recent years, in the field of numerical simulations, the moving-particle semiimplicit (MPS) method has been attracting much interest [1]. The MPS method, one of the particle methods, is the leading numerical-analysis technique that is also capable of treating, and has been used to investigate, complicated 
phenomena such as incompressible inviscid flows with free surfaces and the collapse of a column of water [2]. Advanced materials known as Functionally Graded Materials (FGMs) have drawn considerable interest [3]. These FGMs are expected to be highly heat resistant materials that can be used under high temperature and high temperature gradient conditions. In this case, it is important to investigate the temperature distributions in the FGMs. It is usually difficult to obtain an analytical solution for heat conduction problems with complex geometries and complex thermal boundary conditions. Therefore, a numerical analysis technique is very important. Sladek proposed a method for transient heat conduction analysis in FGMs using the meshless local boundary integral equation [4]. Also Wang developed a meshless numerical method to analyze problems of transient heat conduction in FGMs [5]. Hamza-Cherif solved transient temperature fields in axisymmetric FGM cylinders under various boundary conditions using an h-p finite element method [6]. Dai discussed different material thermal property functions affecting temperature distributions in two-dimensional FGMs using the method of lines [7].

To the best of the author's knowledge, no numerical solutions for heat conduction problems in FGMs by the particle method have been reported. The purpose of this our investigation is to perform analysis of transient and steadystate heat conduction analysis of two-dimensional FGMs using the particle method. In this work, the thermal conductivity or the thermal diffusion coefficient of FGMs is a function of the spatial coordinates. Moreover, although a particle method belongs to the category of meshless methods, the formulations for numerical analysis are mathematically simple, unlike the integral equation method. First, the basic discrete equations for transient and steady-state heat conduction analysis of FGMs are derived. For a few numerical examples, analytical solutions and finite element solutions are compared with the present results. The effects of the radius of interaction and number of particles are also discussed. We obtained good agreement between our present results and those of others with respect to the temperature values and temperatures distributions. This agreement shows the validity of the present method.

\section{Heat conduction equation in FGMs}

In the Cartesian co-ordinate system, the two-dimensional equation governing the heat conduction problem of FGMs without internal heat generation is given by eq. (1) or eq. (2),

$$
\rho c \frac{\partial T}{\partial t}=\nabla \cdot(\lambda \nabla T)
$$

or

$$
\frac{\partial T}{\partial t}=\nabla \cdot(D \nabla T)
$$


where, $t$ is time, $T=T(x, y)$ is the temperature function, and $\lambda=\lambda(x, y)$ denotes the thermal conductivity and is a function of spatial coordinates, $\rho=\rho(x, y)$ is the mass density, $c=c(x, y)$ is the specific heat, and $D(x, y)=\lambda(x, y) / \rho(x, y) c(x, y)$ is the thermal diffusion coefficient respectively.

\section{Discretization of gradient and Laplacian}

From eq. (2), the following expression is obtained

$$
\frac{\partial T}{\partial t}=(\nabla D) \cdot(\nabla T)+D \nabla^{2} T
$$

From the above equation, the discretization of the gradient vectors and Laplacian operator are needed [1,2]. The discretization of the gradient vector of a scalar function $\phi(x, y)$ at the $i$-particle is given by

$$
\langle\nabla \phi\rangle_{i}=\frac{d}{n_{i}} \cdot \sum_{j \neq i}\left[\frac{\phi_{j}-\phi_{i}}{\left|\boldsymbol{r}_{j}-\boldsymbol{r}_{i}\right|^{2}}\left(\boldsymbol{r}_{j}-\boldsymbol{r}_{i}\right) w\left(\left|\boldsymbol{r}_{j}-\boldsymbol{r}_{i}\right|\right)\right]
$$

where $\phi_{i}, \phi_{j}$ are the values of scalar function $\phi(x, y), \boldsymbol{r}_{i}, \boldsymbol{r}_{j}$ are the position vectors of $i$-particle and $j$-particle respectively, $\left|\boldsymbol{r}_{j}-\boldsymbol{r}_{i}\right|$ is the distance between $i$-particle and $j$-particle, $n_{i}$ is the particle density, $d$ is the dimensional number which equals 2 in the two-dimensional problems, the function $w$ is the weighted function and $\Sigma$ means summation with respect to $j \neq i$. The weighted function $w$ is given as follows,

$$
w(r)= \begin{cases}\frac{r_{e}}{r}-1 & \left(0 \leq r<r_{e}\right) \\ 0 & \left(r_{e} \leq r\right)\end{cases}
$$

where, $r$ is the distance between two particles, and $r_{e}$ is the radius of the interaction. Hence, if $r$ is less than $r_{e}$, there is interaction between two particles. The particle density $n_{i}$ is given as follows,

$$
n_{i}=\sum_{j \neq i} w\left(\left|\boldsymbol{r}_{j}-\boldsymbol{r}_{i}\right|\right)
$$

The discretization of the Laplacian operator of the scalar function $\phi(x, y)$ at the $i$-particle is given by 


$$
\left\langle\nabla^{2} \phi\right\rangle_{i}=\frac{2 d}{\Lambda n_{i}} \cdot \sum_{j \neq i}\left[\left(\phi_{j}-\phi_{i}\right) w\left(\left|\boldsymbol{r}_{j}-\boldsymbol{r}_{i}\right|\right)\right]
$$

where, $\Lambda$ is a constant given by the following expression

$$
\Lambda=\frac{\sum_{j \neq i}\left|\boldsymbol{r}_{j}-\boldsymbol{r}_{i}\right|^{2} w\left(\left|\boldsymbol{r}_{j}-\boldsymbol{r}_{i}\right|\right)}{\sum_{j \neq i} w\left(\left|\boldsymbol{r}_{j}-\boldsymbol{r}_{i}\right|\right)} .
$$

\section{Basic discrete equation}

Applying Euler's explicit method to the term on the left hand side of eq. (3), and discretization of the gradient vector and Laplacian operator on the right side, the basic discrete equation for the transient heat conduction can be obtained as follows,

$$
\begin{gathered}
T_{i}^{s+1}=T_{i}^{s}+\Delta t \cdot \sum_{j \neq i} \frac{2 d}{\Lambda n_{i}}\left[D_{i}\left(T_{j}^{s}-T_{i}^{s}\right) \cdot w\left(\left|\boldsymbol{r}_{j}-\boldsymbol{r}_{i}\right|\right)\right] \\
+\Delta t \cdot\left(\begin{array}{l}
\sum_{j \neq i} \frac{d}{n_{i}}\left[\frac{T_{j}^{s}-T_{i}^{s}}{\left|\boldsymbol{r}_{j}-\boldsymbol{r}\right|^{2}}\left(\boldsymbol{r}_{j}-\boldsymbol{r}_{i}\right) \cdot w\left(\left|\boldsymbol{r}_{j}-\boldsymbol{r}_{i}\right|\right)\right] \\
\cdot \sum_{j \neq i} \frac{d}{n_{i}}\left[\frac{D_{j}-D_{i}}{\left|\boldsymbol{r}_{j}-\boldsymbol{r}_{i}\right|^{2}}\left(\boldsymbol{r}_{j}-\boldsymbol{r}_{i}\right) \cdot w\left(\left|\boldsymbol{r}_{j}-\boldsymbol{r}_{i}\right|\right)\right]
\end{array}\right)
\end{gathered}
$$

where the upper subscript $\mathrm{s}$ is the time step number and $\Delta t$ is the time increment. The dot inside the parenthesis of the third term on the right hand side denotes the inner product. The basic discretized simultaneous equation for the steady state heat conduction is given by,

$$
\begin{aligned}
& \sum_{j \neq i} \frac{2 d}{\Lambda n_{i}}\left[D_{i}\left(T_{j}-T_{i}\right) \cdot w\left(\left|\boldsymbol{r}_{j}-\boldsymbol{r}_{i}\right|\right)\right] \\
& +\left(\begin{array}{l}
\sum_{j \neq i} \frac{d}{n_{i}}\left[\left(T_{j}-T_{i}\right)\left(\boldsymbol{r}_{j}-\boldsymbol{r}_{i}\right) \frac{w\left(\left|\boldsymbol{r}_{j}-\boldsymbol{r}_{i}\right|\right)}{\left|\boldsymbol{r}_{j}-\boldsymbol{r}_{i}\right|^{2}}\right] \\
\left.\cdot \sum_{j \neq i} \frac{d}{n_{i}}\left[\left(D_{j}-D_{i}\right)\left(\boldsymbol{r}_{j}-\boldsymbol{r}_{i}\right) \frac{w\left(\left|\boldsymbol{r}_{j}-\boldsymbol{r}_{i}\right|\right)}{\left|\boldsymbol{r}_{j}-\boldsymbol{r}_{i}\right|^{2}}\right]\right)
\end{array}\right)=0
\end{aligned}
$$




\section{Numerical examples}

In order to demonstrate the efficiency and accuracy of the proposed particle method, we first considered steady-state heat conduction problems, and then transient heat conduction in FGMs.

\subsection{Steady-state analysis}

In the first examples, a square FGM is considered as shown in Fig.1. On both opposite sides parallel to the $x$-axis, heat insulation conditions are imposed, and on the lateral sides, two different temperatures are prescribed. Initial temperature of the whole region is zero.

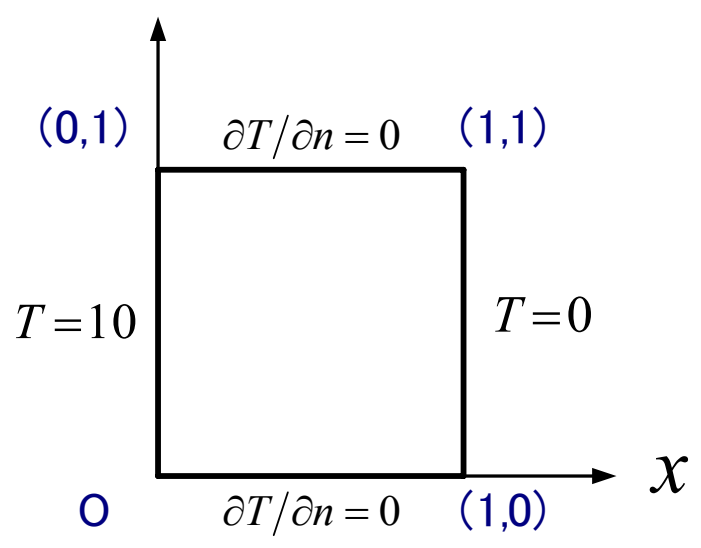

Figure 1: Geometry and boundary conditions.

In numerical calculations, 11 particles are located along $x$-axis and $y$-axis respectively for a total of 121 particles. The radius of interaction $r_{e}$ in eq. (5) is twice the minimum distance between two particles.

Particles on the boundary have interaction between only inner particles, no prescribed temperatures are imposed and heat insulation conditions are satisfied automatically.

\subsubsection{Example 1}

The distribution of thermal conductivity of the FGM is assumed as,

$$
\lambda(x)=A \exp (B x),
$$

where $\lambda$ is the thermal conductivity and $A$ and $B$ are constants [8]. In case $A=1, B=-2,0,2$, the distribution of the thermal conductivity is shown in Fig.2. The case of $B=0$ corresponds to an isotropic material. 


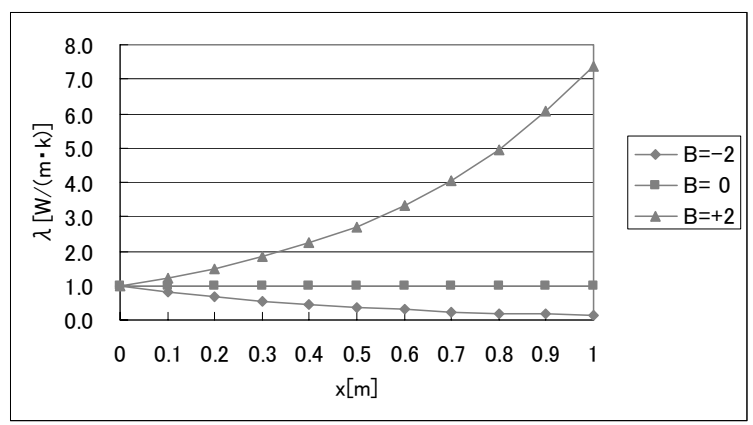

Figure 2: $\quad$ Thermal conductivity of eq. (11).

The temperature distributions simulated by the present method and exact solutions along the line $y=0.5$ in FGM are shown in Fig.3 [8]. For each value of constant $B$, the numerical results are in good agreement with the analytical solutions.

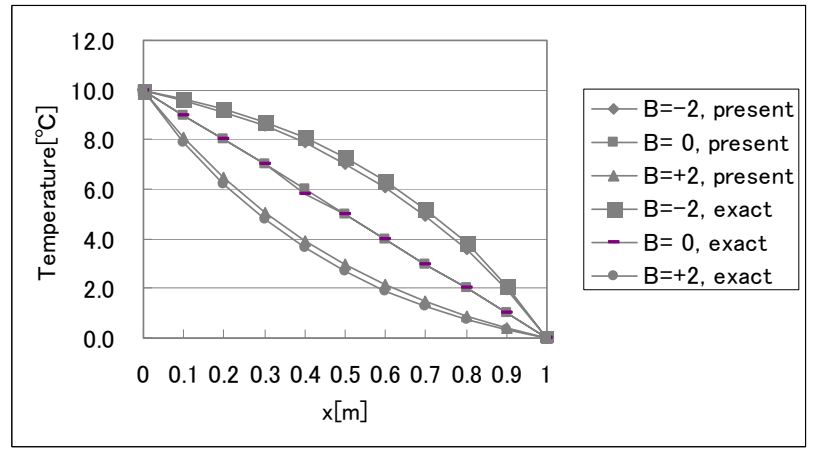

Figure 3: Distribution of steady-state temperature.

\subsubsection{Example 2}

Thermal conductivity in the material changes according to the following equation,

$$
\lambda(x, y)=1+2 \sin \left(\frac{\pi x}{L}\right) \sin \left(\frac{\pi y}{L}\right)
$$

where the side-length of the square is $L=1 \mathrm{~m}$ [8]. In this material, the thermal conductivity changes in $x$-axis and $y$-axis directions. The distribution of the thermal conductivities along $y=0.1$ and $y=0.5$ are presented in Fig.4. The temperature distribution obtained by the present method and a FEM solution along the line $y=0.5$ are presented in Fig.5 [8]. In this case, both are in good agreement. 


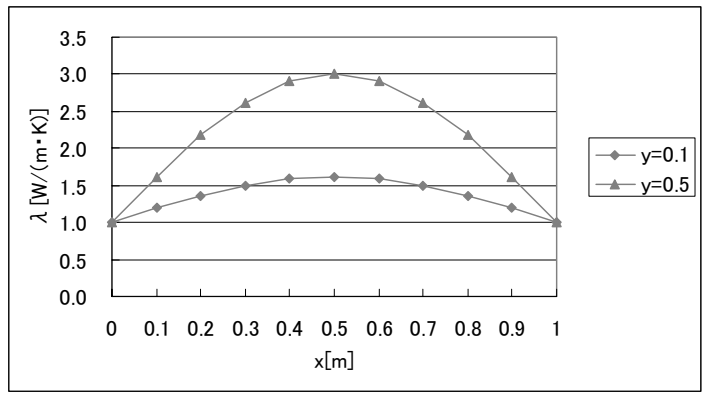

Figure 4: Thermal conductivity of eq. (11).

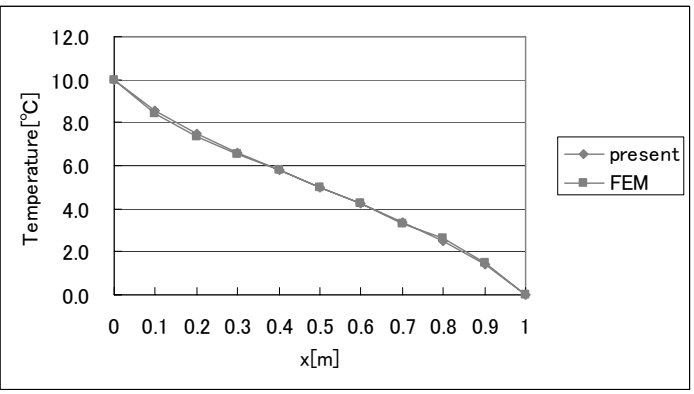

Figure 5: Distribution of steady-state temperature.

\subsection{Transient analysis}

Carrying out the transient simulation until the steady state is achieved, the results are compared with analytical solutions obtained by Fourier transform.

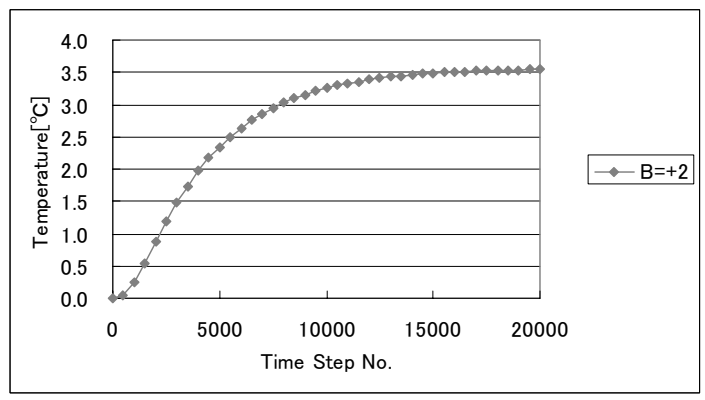

Figure 6: Time variation of temperature.

In the numerical calculations we, for convenience, use a square with sidelength $L=1 \mathrm{~m}$, take the thermal conductivity as $B=2$ (eq. (11)) and the thermal conductivity $\rho c=1000$. Take 11 particles along $x$-axis and $y$-axis respectively so a total of 121 particles are used. The radius of interaction in eq. (5) is twice 
the minimum distance between two particles and $\Delta t=1.0 \times 10^{-2} \mathrm{~s}$ time increment is used. The variation of temperature at the central point $(0.5,0.5)$ is presented in Fig.6, and the field of the stationary temperature along $y=0.5$ at time step 20,000 with the analytical solution of steady-state is shown in Fig.7. Because of the convenient thermal capacity, these results have no physical meaning. The temperature reached steady-state at about 15,000 time steps and there was excellent agreement between the results of the proposed method and the exact solution by steady-state analysis.

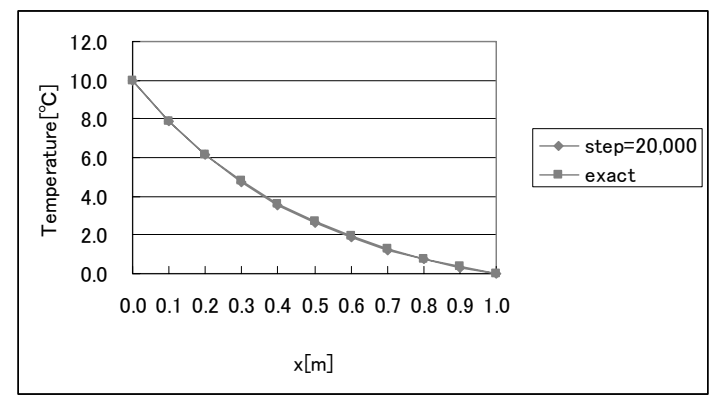

Figure 7: Stationary temperature distribution.

\subsection{Effect of number of particles}

In this section, number of particles affecting the accuracy of the steady-state solutions is examined. The thermal conductivities of eq.(11) with $B=2$ and eq. (12) are analyzed. The geometry, boundary conditions, and the interaction radius of the problem are the same as above. The temperature distributions along $y=0.4$ under eq.(11) analysis using $6 \times 6,11 \times 11$, and $16 \times 16$ particles respectively are shown in Fig. 8 . In each case, the accuracies of the results are guaranteed even for the $6 \times 6$ particles.

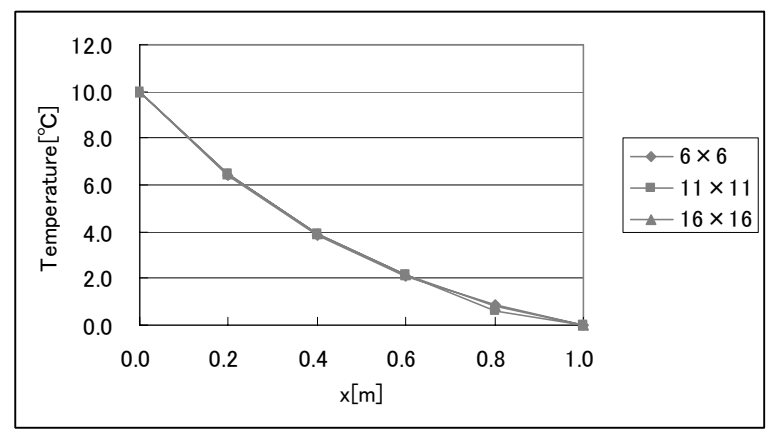

Figure 8: Relationships between temperature and number of particles under eq. (11). 


\subsection{Effect of radius of interaction}

Via parameter analysis with respect to the radius of interaction in the weighted function eq. (5), the influence of these radii on the accuracies of the steady-state results was investigated. The geometry, the distribution of thermal conductivity and boundary conditions is the same as in the above section. Figure 9 shows the relationships between the temperature distributions along $y=0.5$ and the radius of interaction for variation from 5 times to twice the minimum distance between two particles.

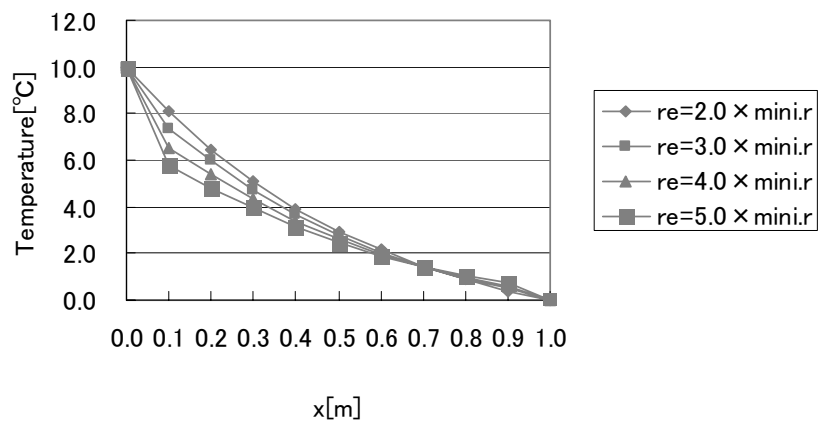

Figure 9: Relationships between temperature distributions and radius of interaction under eq.(11).

Accuracy in the case where the interaction radius is twice the minimum distance between two particles is good, but accuracy falls off with increase in the interaction radius. In reference [1], radius of interaction from 4 times to twice the minimum distance between two particles is recommended.

\section{Conclusion}

In this paper, the transient and steady-state analysis of heat conduction for FGMs using the particle method is presented. The basic discrete equations for heat conduction analysis in FGMs are formulated by the discretizations of gradient and Laplace operators in the heat conduction equation. Solving a few twodimensional problems in FGMs, most of the results correlate well with the exact solutions or those obtained by the finite element method and the validity of the present method is shown. And also, according to the parameter analysis with respect to the number of particles and the radius of interaction in the weighted function, accurate solutions are obtained for even a comparatively small number of particles, and the recommended interaction radius is twice the minimum distance between two particles. 


\section{References}

[1] Koshizuka, S. and Oka, Y., Moving-Particle Semi-implicit Method for Fragmentation of Incompressible Fluid, Nucl. Sci. Eng., 123, pp.421434,1996

[2] Ataie-Ashtiani, B. and Farhadi, L., A stable moving-implicit method for free surface flows, Fluid Dynamics Research, 38, pp.241-256, 2006.

[3] Koizumi, M., FGM activities in Japan, Composites Part B, 288, pp.1-4, 1997.

[4] Sladek, J., Sladek V. and Zhang Ch., Transient heat conduction analysis in functionally graded materials by the meshless local boundary integral equation method, Computational Materials and Sciences, 28, pp.494$504,2003$.

[5] Wang, H., Qin, Q-H. and Kang, Y-L, A meshless model for transient heat conduction in functionally graded materials, Comput Mech , 38, pp.51$60,2006$.

[6] Hamaza-Cherif, S., Houmat, A. and Hadjou, A., TRANSIENT HEAT CONDUCTION IN FUNCTIONALLY GRADED MATERIALS, International Journal of Computational Methods, Vol.4, No.4, pp.603-619, 2007.

[7] Dai, Y., Tan, W. and Li, Y.D., Effect of different thermal conductivity functions on temperature fields in FGM, Journal of Materials Processing Technology, 187-188, pp.212-214, 2007.

[8] Ochiai, Y., Two-dimensional steady heat conduction in functionally gradient materials by triple-reciprocity boundary element method, Engineering Analysis with Boundary Elements, 28, pp.1445-1453, 2004. 